\title{
Plasmonic Color Laser Printing inside Transparent Gold NanodiskEmbedded Poly(dimethylsiloxane) Matrices
}

\author{
Cui, Ximin; Zhu, Xiaolong; Shao, Lei; Wang, Jianfang; Kristensen, Anders
}

Published in:

Advanced Optical Materials

Link to article, DOI:

10.1002/adom.201901605

Publication date:

2020

Document Version

Peer reviewed version

Link back to DTU Orbit

Citation (APA):

Cui, X., Zhu, X., Shao, L., Wang, J., \& Kristensen, A. (2020). Plasmonic Color Laser Printing inside Transparent Gold NanodiskEmbedded Poly(dimethylsiloxane) Matrices. Advanced Optical Materials, 8(1), [1901605]. https://doi.org/10.1002/adom.201901605

\section{General rights}

Copyright and moral rights for the publications made accessible in the public portal are retained by the authors and/or other copyright owners and it is a condition of accessing publications that users recognise and abide by the legal requirements associated with these rights.

- Users may download and print one copy of any publication from the public portal for the purpose of private study or research.

- You may not further distribute the material or use it for any profit-making activity or commercial gain

- You may freely distribute the URL identifying the publication in the public portal 


\title{
Plasmonic Color Laser Printing inside Transparent Gold Nanodisk-Embedded Poly(dimethylsiloxane) Matrices
}

Ximin Cui, Xiaolong Zhu, Lei Shao, Jianfang Wang, * and Anders Kristensen*

Dr. X. M. Cui, Prof. L. Shao, Prof. J. F. Wang

Department of Physics

The Chinese University of Hong Kong

Shatin, Hong Kong SAR, China

E-mail: jfwang@phy.cuhk.edu.hk

Dr. X. L. Zhu, Prof. A. Kristensen

DTU Health Tech

Department of Health Technology

Technical University of Denmark

DK-2800 Kongens Lyngby, Denmark

E-mail: akri@dtu.dk

Keywords: gold nanodisks, laser color printing, plasmonic colors, plasmons, poly(dimethylsiloxane)

\begin{abstract}
Plasmonic color generation from metal nanostructures has attracted intensive attention because of their excellence in achieving high spatial resolution, strong color contrast and long-term durability. The limited area of plasmonic patterns anchored on substrates and produced by current top-down methods, however, severely restricts the advanced developments and potential applications in structural color display. Herein we present a robust method for realizing the laser printing of plasmonic colors inside transparent gold nanodiskembedded poly(dimethylsiloxane) matrices. We find that various colors can not only be easily generated by embedding gold nanodisks of different sizes, but also finely varied by adjusting the laser pulse intensity during printing. We further demonstrate that multiple color layers can be laser-printed at different depths. Stereoscopic images in the three-dimensional matrices are laser-printed with sizes as large as $12 \times 15 \mathrm{~mm}^{2}$ and a resolution of 4600 dots per inch.
\end{abstract}

Color generation from noble metal nanostructures links ancient Roman dichroic glass craftsmanship to rapidly emerging nanophotonic color technologies of today. ${ }^{[1-3]}$ Noble metal 
nanostructures possess the advantages of confining light at nanoscale, generating tailorable spectroscopic responses, and exhibiting extremely large scattering and absorption crosssections because of their localized surface plasmon resonances (LSPRs). ${ }^{[4,5]}$ Plasmonic colors can therefore have high spatial resolution, high color contrast, and long-term durability. ${ }^{[6-8]}$ These properties have served as the base for numerous color-related applications, such as subwavelength-resolution color printing, ${ }^{[6,9-11]}$ macroscopic color holograms,${ }^{[12,13]}$ dynamic color display, ${ }^{[14,15]}$ and full-color filters and polarizers. ${ }^{[16,17]}$

Plasmonic colors can be generated by designing or postprocessing metal nanostructures. Lithographic patterning ${ }^{[6,18,19]}$ and focused ion beam milling ${ }^{[20,21]}$ can yield nanostructure color dots with predesigned geometries at relatively high cost, while laser printing ${ }^{[22,23]}$ is a cost-effective postprocessing technique that can allow for the tuning of plasmonic colors produced by imprinting. We demonstrated in our previous work a resonant laser postprinting method for generating tunable structural colors through the melting and reshaping of largescale imprinted nanostructures. ${ }^{[10,22]}$ However, both predesigned and imprinted nanostructures need to be supported on substrates, forming two-dimensional architectures. The laser pulse rapidly melts the nanostructures into spherical nanoparticles, which experience repulsive momenta caused by the photons, resulting in random offsets on the substrates during laser printing. ${ }^{[22,24,25]}$ In addition, metal nanostructures prepared by the aforementioned top-down fabrication techniques usually suffer from large plasmon damping caused by their polycrystalline nature and the use of adhesive layers. ${ }^{[26,27]}$ The manufacturing of plasmonic nanostructures usually involves multiple steps of nanofabrication that is of high cost and time consumption and is challenging to scale up. ${ }^{[28,29]}$ A robust method is therefore highly desired for the fabrication of plasmonic color patterns in three-dimension (3D) matrices.

The chemical growth of colloidal metal nanocrystals in solutions is very promising for the low-cost mass production of crystallographically well-defined nanostructures. Through the chemical reduction of metal salts in solutions, metal nanocrystals are grown homo- or 
hetero-epitaxially and therefore possess good crystallinity, high morphological uniformity as well as good tunability in size and shape. ${ }^{[5,30,31]}$ Among various nanocrystals, Au nanodisks (NDs) are ideal building blocks for plasmonic color generation owing to their circular symmetry and large spectral tunability. Circular Au NDs possess a single plasmon resonance mode and exhibit polarization independence in their disk plane, endowing them with a predictable and tailorable color rendering. ${ }^{[32,33]}$ Compared with spherical or rod-like equivalents composed of a similar quantity of $\mathrm{Au}$ atoms, circular $\mathrm{Au}$ NDs possess larger scattering and absorption cross-sections at their resonance wavelength, which is beneficial to the generation of bright colors. ${ }^{[34,35]}$ It has been challenging, however, to manipulate unanchored nanocrystals in solutions precisely or assemble them into ordered arrays. Colloidal metal nanocrystals incline to aggregate during the drying of solutions, resulting in the change and uncontrollability of their plasmonic colors. To address the above challenges, colloidal metal nanocrystals can be functionalized and embedded into poly(dimethylsiloxane) (PDMS) to preserve their original plasmonic colors. ${ }^{[36,37]}$ PDMS is one of the widely employed polymers in micro- and nanotechnologies for its outstanding properties of flexibility, optical transparency and ease of large-scale production. ${ }^{[38-41]}$ The use of PDMS can not only retain the optical properties of plasmonic metal nanocrystals thoroughly, but also protect them from greasy residues, mechanical damage and fingerprints successfully. These excellent properties enable fabricated, nanocrystal-embedded PDMS matrices to function as an ideal platform for the manipulation of metal nanocrystals and further engineering of their plasmonic colors.

In this work, we show that plasmonic color patterns can be robustly laser-printed in 3D PDMS matrices embedded with circular Au NDs. PDMS matrices with different colors are fabricated by embedding colloidal circular Au NDs of various diameters. Single laser pulses are employed to melt and reshape the embedded NDs through local heat generation. Tunable plasmonic colors are readily generated from the reshaped NDs by adjusting the laser pulse 
energy density. As demonstration, different optical images at the centimeter scale are successfully laser-printed in the circular Au ND-embedded PDMS matrices. Taking advantage of the transparent nanocrystal-embedded PDMS matrices, the position of the focused laser spot has access to a 3D space and is no longer limited to a particular surface, which allows for the laser printing of plasmonic color patterns at different depths. In our experiments, three layers with different colors are laser-printed at different depths, suggesting a potential for creating 3D optical images by printing more layers. Our printing approach has the advantages of low cost, large area, and durability for practical applications.

The circular Au ND-embedded PDMS matrices are prepared for the laser printing of plasmonic colors, as illustrated schematically by the flowchart in Figure 1. The circular Au nanodisks are chemically synthesized and stably dispersed in aqueous solutions, using cetyltrimethylammonium bromide (CTAB) as the stabilizing and structure-directing agent. To retain their plasmonic properties for further laser patterning, the Au NDs are re-dispersed homogeneously in PDMS prepolymer, which is thereafter polymerized to produce the $\mathrm{Au}$ ND-embedded PDMS matrices. Under the exposure of a pulsed laser, the instant photothermal conversion of the irradiated NDs generates a high enough local temperature to melt and reshape the Au NDs. The shape and size of the resultant nanocrystals, which significantly alter the frequency of their LSPRs, are highly dependent on the input pulse energy density. The LSPRs of the reshaped nanocrystals are therefore readily controlled during the morphology transformation, enabling the laser printing of finely tunable plasmonic colors. Furthermore, the cuboid PDMS matrices allow for a stereo scanning of the laser spot for the fabrication of plasmonic color patterns at different depths. 


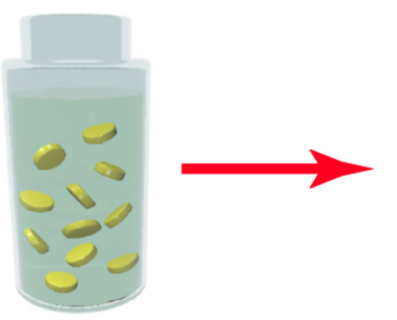

Au nanodisk solution

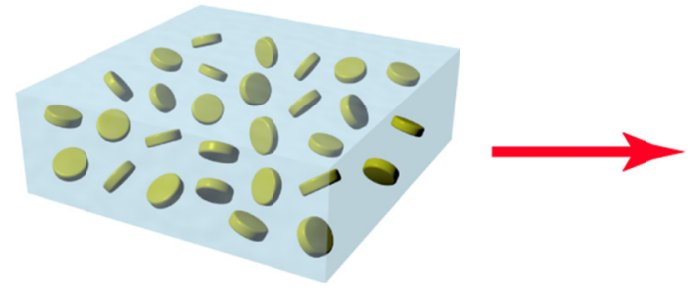

Au nanodisk-embedded PDMS matrix

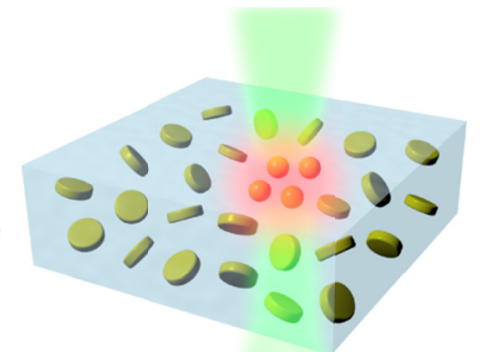

plasmonic laser printing

Figure 1. Flowchart of the plasmonic color printing. The schematics of the aqueous Au ND solution (left), the Au ND-embedded PDMS matrix (middle), and the laser printing of the Au ND-embedded PDMS matrix (right) are shown. The Au NDs can be thermally reshaped into nanospheres under single-pulse laser exposure with sufficient pulse energy.

We synthesized Au nanoplates by seed-mediated growth and performed subsequent anisotropic oxidation to produce circular Au NDs with different in-plane dipolar resonance wavelengths. ${ }^{[32,42]}$ During oxidation, the sharp corners of the as-grown Au nanoplates first become obtuse and gradually transform into round edges. The diameter of the formed Au NDs can be finely reduced by simply increasing the oxidation time. By varying the oxidation duration, we prepared one obtuse Au nanoplate sample and five circular Au ND samples (Figure 2a). The average edge sides or diameters of the six oxidized samples determined from the scanning electron microscopy (SEM) images are $131 \pm 13 \mathrm{~nm}, 98 \pm 8 \mathrm{~nm}, 84 \pm 6 \mathrm{~nm}, 74 \pm$ $6 \mathrm{~nm}, 70 \pm 5 \mathrm{~nm}$ and $68 \pm 6 \mathrm{~nm}$, respectively. Oxidation selectively occurs at the sharp corners and side edges because of the enrichment of iodide ions on the top and bottom surfaces of the as-grown Au nanoplates. ${ }^{[32,34]}$ As a result, the obtuse Au nanoplates and circular Au NDs possess a constant thickness of $17.4 \pm 1.1 \mathrm{~nm}$, which was confirmed from the height profiles acquired with an atomic force microscope (AFM) (Figure S1, Supporting Information). Because these $\mathrm{CTAB}$-stabilized $\mathrm{Au}$ NDs rapidly aggregate upon the direct dispersion into the PDMS prepolymer, surfactant replacement was performed from CTAB to thiol-terminated methoxy poly(ethylene glycol) (mPEG-SH). ${ }^{[36]}$ The PEGylated Au NDs were homogeneously dispersed in the PDMS prepolymer, which was thereafter thermally 
polymerized to form the circular Au ND-embedded PDMS matrices (Figure 2b). The PDMS

matrices exhibit uniform colors and remain transparent after the embedding of the circular $\mathrm{Au}$ NDs. As the particle number remained unchanged during oxidation, the six ND samples have the same particle concentration, and so do their matrices. The initial colors of the aqueous $\mathrm{Au}$ ND solutions are therefore successfully preserved by embedding the Au NDs into the PDMS matrices.
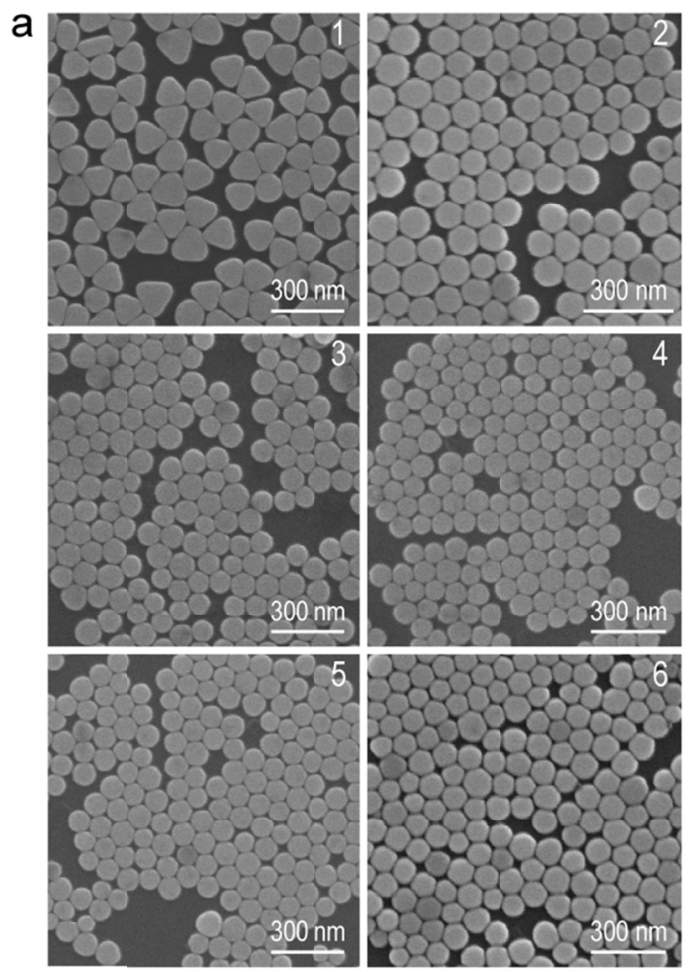
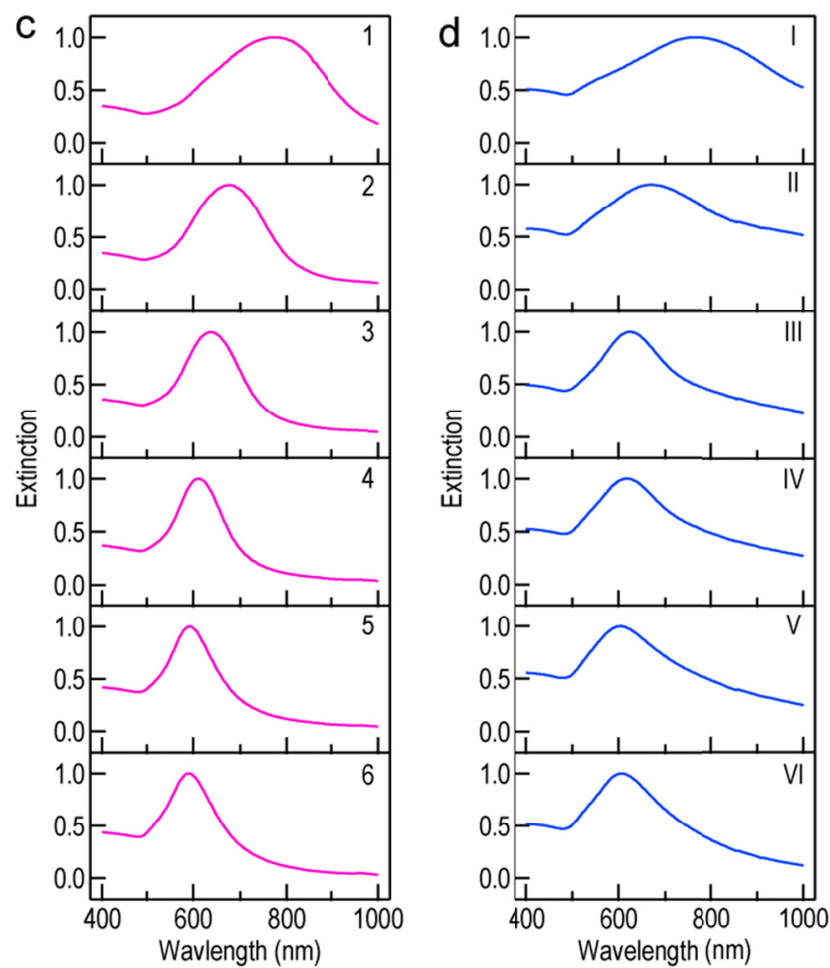

b

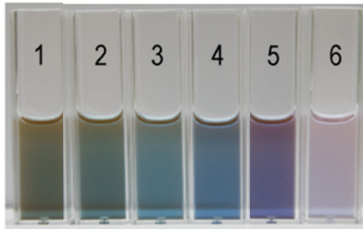

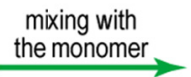

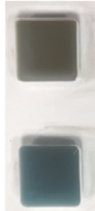

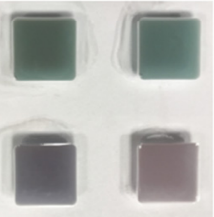

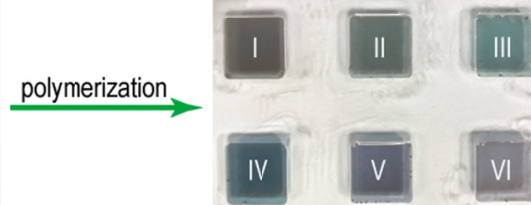

Figure 2. Embedment of the Au NDs in PDMS. a) SEM images of the Au NDs with different diameters. The six Au ND samples are labeled as 1-6, with their average edge sides or diameters being $131 \pm 13 \mathrm{~nm}, 98 \pm 8 \mathrm{~nm}, 84 \pm 6 \mathrm{~nm}, 74 \pm 6 \mathrm{~nm}, 70 \pm 5 \mathrm{~nm}$ and $68 \pm 6 \mathrm{~nm}$, respectively. b) Photographs of the aqueous Au ND solutions (left), the PDMS prepolymer dispersions of the differently sized Au ND samples (middle), and the Au ND-embedded PDMS matrices (right). The matrices I-VI correspond to the Au ND samples 1-6 as embedded in PDMS. Each PDMS matrix has a side length of $\sim 2 \mathrm{~cm}$ and a thickness of $\sim 0.5$ $\mathrm{cm}$. c,d) Measured extinction spectra of the aqueous Au ND samples 1-6 and the Au NDembedded PDMS matrices I-VI, respectively. 
The extinction spectra (Figure 2c) show that the in-plane dipolar plasmon resonance of the aqueous $\mathrm{Au}$ ND samples is centered in the visible range from $\sim 600 \mathrm{~nm}$ to $\sim 800 \mathrm{~nm}$. The plasmon peak exhibits a controllable blueshift as the ND diameter is reduced, which was also verified by the finite-difference time-domain (FDTD) simulations (Figure S2, Supporting Information). Because of the intrinsic property of the circular symmetry, the excitation energy is completely pumped into a single plasmon mode, resulting in the observation of the sole plasmon resonance. The size fluctuation in the chemical growth leads to a broadening of the extinction peaks of the nanocrystal solutions in comparison with the simulation results performed for the individual nanocrystals. The plasmon resonance wavelengths of the $\mathrm{Au}$ NDs embedded in the PDMS matrices are very close to those in the aqueous solutions (Figure 2d). Slight redshifts were observed owing to the refractive index change of the surrounding environment (Figure S3, Supporting Information). The observed peak broadening results from the slight aggregation of the Au NDs in PDMS. Because of the pseudo-two-dimensional nature, the disk-like Au nanocrystal exhibits a much larger scattering and absorption crosssection at its plasmon resonance wavelength than the Au nanosphere or nanorod with the same particle volume (Figure S4, Supporting Information). Bright and tunable colors can therefore be generated in the transparent PDMS matrices by incorporating Au NDs with different plasmon resonance wavelengths.

After being embedded in the PDMS matrices to yield different vivid colors, the Au NDs can be further treated with laser irradiation to alter their plasmon resonances and therefore generate different colors. We employed single laser pulses $(1 \mathrm{~ns}, \lambda=532 \mathrm{~nm})$ to melt and reshape the Au NDs in the PDMS matrices. There exists a threshold value for the laser irradiation energy where the melting reaction is initiated. ${ }^{[22,43]}$ Because of the Gaussian density distribution of the laser energy profile, only the Au NDs in the central region of the laser spot can transiently accumulate sufficient energy to get melted under the irradiation of one laser pulse. The morphology of the reshaped NDs can be controlled by adjusting the input 
energy of the laser pulse, enabling laser printing to generate controllable colors. Owing to the subwavelength light confinement of LSPRs, the laser-induced melting can be localized at the targeted Au NDs, enabling a high spatial resolution of plasmonic laser printing. ${ }^{[6,44]}$ In addition, the highly asymmetric shape of the Au NDs, which can magnify the LSPR spectral change when the Au atoms migrate from the high to low curvature sites during the melting process, is beneficial for the color printing since a low threshold of the laser pulse energy is required to induce a significant LSPR shift.

Figure 3a shows a photograph of the Au ND-embedded PDMS matrix I that was printed to exhibit various colors from magenta to yellow. The pulse energy applied on the color cells i-vi are $2.5 \mathrm{~nJ}, 0.15 \mu \mathrm{J}, 0.6 \mu \mathrm{J}, 1.3 \mu \mathrm{J}, 2.2 \mu \mathrm{J}$ and $3.0 \mu \mathrm{J}$, respectively. As focused through a $4 \times$ objective lens (numerical aperture: 0.13 ), the laser pulse has a spot size of $\sim 50 \mu \mathrm{m}$ in diameter, which is much smaller than the matrix thickness of $\sim 0.5 \mathrm{~cm}$. Only a thin layer of the $\mathrm{Au}$ NDs in the PDMS matrix was thermally melted under the laser irradiation. As a result, the reshaped ND layer together with the background layer of the unchanged NDs collectively contributes to the observed colors. The extinction spectra of the color cells i-vi in Figure $3 \mathrm{~b}$ were measured using a black mask with a pinhole of $2 \times 2 \mathrm{~mm}^{2}$, which has the same size as each cell. As printed with the laser irradiation at a relatively low intensity, the color cell $\mathrm{i}$ exhibits two comparable plasmon resonance peaks at 580 and $770 \mathrm{~nm}$. The two peaks have comparable extinction intensities. They are originated from the printed and background ND layers, respectively. As the laser pulse energy is increased, the plasmon peak of the reshaped NDs gradually blueshifts. We further used the matrices I-VI with different background colors to explore the fine tuning of the laser-printed plasmonic colors in the visible region (Figure S5, Supporting Information). The CMY primaries (cyan, magenta and yellow) can remarkably be obtained through the plasmonic color printing in the Au ND-embedded PMDS matrices with different background colors. 

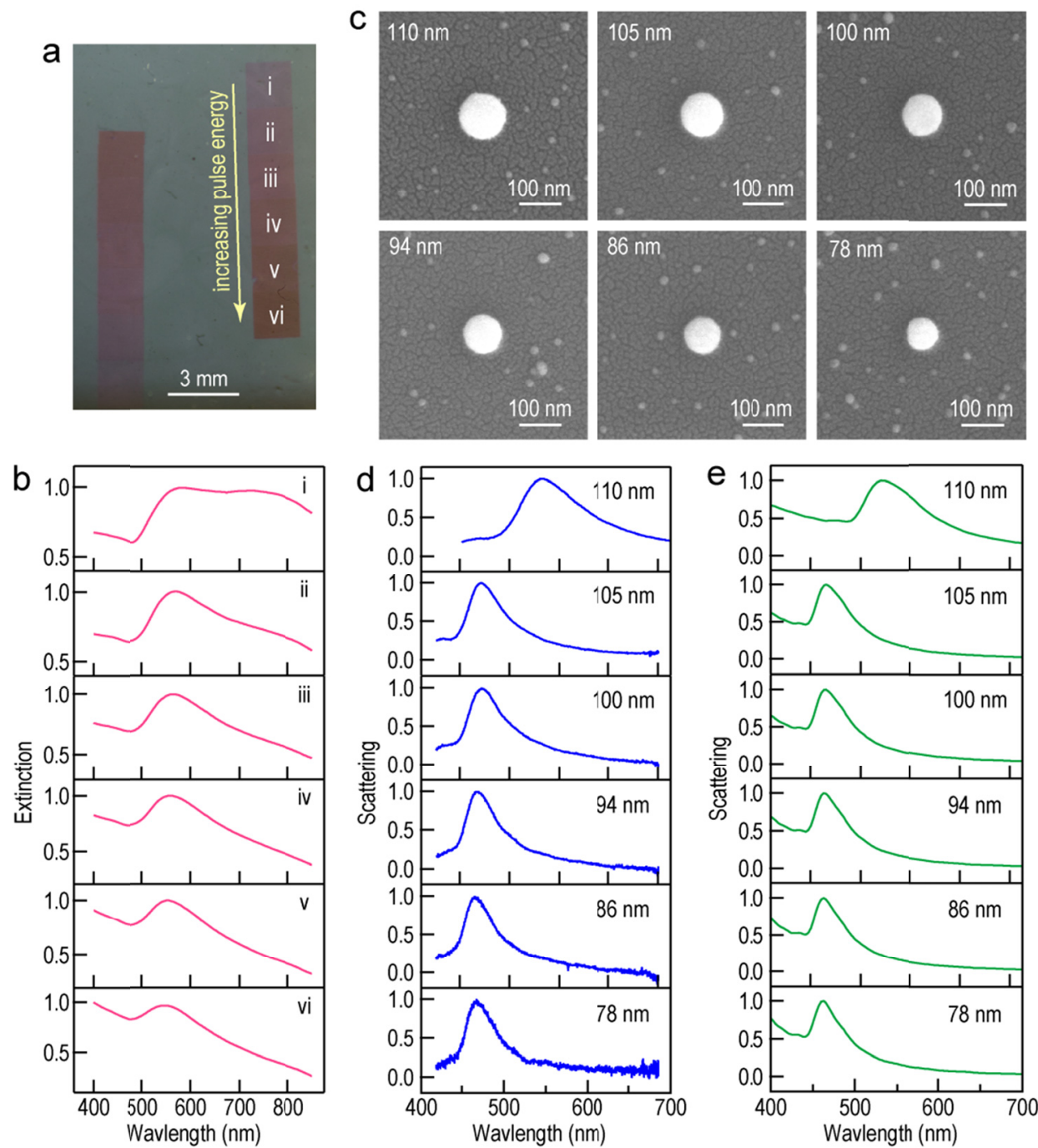

Figure 3. Spectral and geometry analysis of the plasmonic color printing. a) Photograph of the Au ND-embedded PDMS matrix I with the laser-printed color cells i-vi generated by increasing the laser pulse energy. b) Measured extinction spectra of the corresponding color cells in (a). c) Representative SEM images of the Au spheroidal nanoparticles produced by the laser irradiation of the glass-supported Au NDs with different pulse energies. The laserirradiated Au ND sample is the same as that in (a). The number at the upper left of each image represents the particle diameter. d) Measured scattering spectra of the corresponding nanoparticles in (c). e) Corresponding simulated scattering spectra.

The Au NDs are randomly embedded in the nonconductive PDMS matrices. Their morphological change caused by the laser irradiation is difficult to be examined directly. Considering the close thermal conductivity between cover glass and PDMS polymer, we investigated the morphological change by performing the laser printing on the glass-supported $\mathrm{Au}$ NDs under the same conditions. Figure 3c shows the representative SEM images of the single reshaped Au NDs after the laser irradiation with different pulse energies. Because of 
the plasmonic photothermal effect, the Au NDs can be readily melted at their rims and transformed into spheroidal nanoparticles with low energy consumption. By further increasing the laser pulse energy, the reshaped NDs can be gradually ablated and reduced in diameter because of the repulsive momentum in the melting process. ${ }^{[22,24,43]}$ The sequential change of the particle shape and size leads to a controllable color printing by simply varying the laser pulse energy. Before the glass-supported nanoparticles were sputter-coated with Au for SEM imaging, we acquired the single-particle scattering spectra of the reshaped NDs produced by the laser treatment (Figure 3d). The gradual decrease in the diameter of the spheroidal nanoparticles leads to the blueshift of their scattering peaks, which has a qualitative agreement with the extinction spectra in Figure 3b. The simulated results in Figure 3e also verify the blueshift of the scattering peaks. Because of the presence of the untreated NDs and the different refractive indexes of the surrounding environments, the ensemble reshaped NDs in the PDMS matrices show a slightly different spectral feature from the single ones on the cover glass substrates.

To illustrate the capability of our plasmonic laser printing, we printed several large-area stereoscopic images with plasmonic color control in the Au ND-embedded PDMS matrices. Figure 4a shows the photograph of a laser-printed facial design of Peking opera. The original photograph for the laser printing was first converted into a grayscale image, in which the digital brightness of each pixel represents the laser pulse energy value. An image of the facial design was therefore laser-printed with a size as large as $12 \times 15 \mathrm{~mm}^{2}$ (Figure $4 \mathrm{a}$ ) according to the mapping relationship between the printed colors and the laser pulse energy in Figure 3a and b. Our plasmonic laser printing technology can be scaled up as long as larger-area PDMS matrices embedded with homogeneous Au NDs are fabricated. By applying the Au NDembedded PDMS matrices with different background colors, the badge of Technical University of Denmark was printed in various color tones (Figure 4b). More images printed in the different PDMS matrices are shown in Figure S6 (Supporting Information). They were all 
captured with a commercial digital camera (Nikon D7000). Laser printing with plasmonic nanoparticles offers a powerful means for achieving the sub-diffraction-limit resolution. ${ }^{[10,22]}$ The localized electromagnetic field enhancement of the nanoparticle together with the modulation of the Gaussian-like laser beam enables a small region to reach the melting threshold. Plasmonic laser printing can therefore be realized at the single-particle level. The printing resolution is eventually limited by the distance among neighboring particles in the PDMS matrix. As mentioned above, the PDMS matrices were fabricated with the same particle density of $6.0 \times 10^{15} \mathrm{~m}^{-3}$, which corresponds to an average particle spacing of $\sim 5.5$ $\mu \mathrm{m}$. As a result, all images were printed at a resolution of 4600 dots per inch (DPI). Furthermore, the feasibility of the traditional halftoning technology was demonstrated using our plasmonic laser printing technology. Figure $4 \mathrm{c}$ shows the reflection and transmission stereoscopic halftoned images of Mona Lisa. Two Au ND-embedded PDMS matrices IV were laser-printed with the complementary images by reversely encoding the grayscale intensities. The reverse encoding means that each pixel in the negative image was printed by the reversed laser pulse energy of the corresponding pixel in the positive image. Interestingly, the positive and negative images can be converted to each other by altering the incident light because of the LSPR properties of the Au NDs. This property endows the Au ND-embedded PDMS matrices with the chromotropic capability under reflected and transmitted light. When the printed transparent PDMS matrices are placed in front of a white background, the reflective images exhibit a mixture of the magenta and cyan colors. As the white background is replaced by a black one, the magenta color turns cyan-yellow for the images and the cyan color becomes yellow for the PDMS matrices. In addition, the stereoscopic images with the printed CMY colors can be preserved in these transparent, flexible and hydrophobic PDMS matrices for more than six months. 

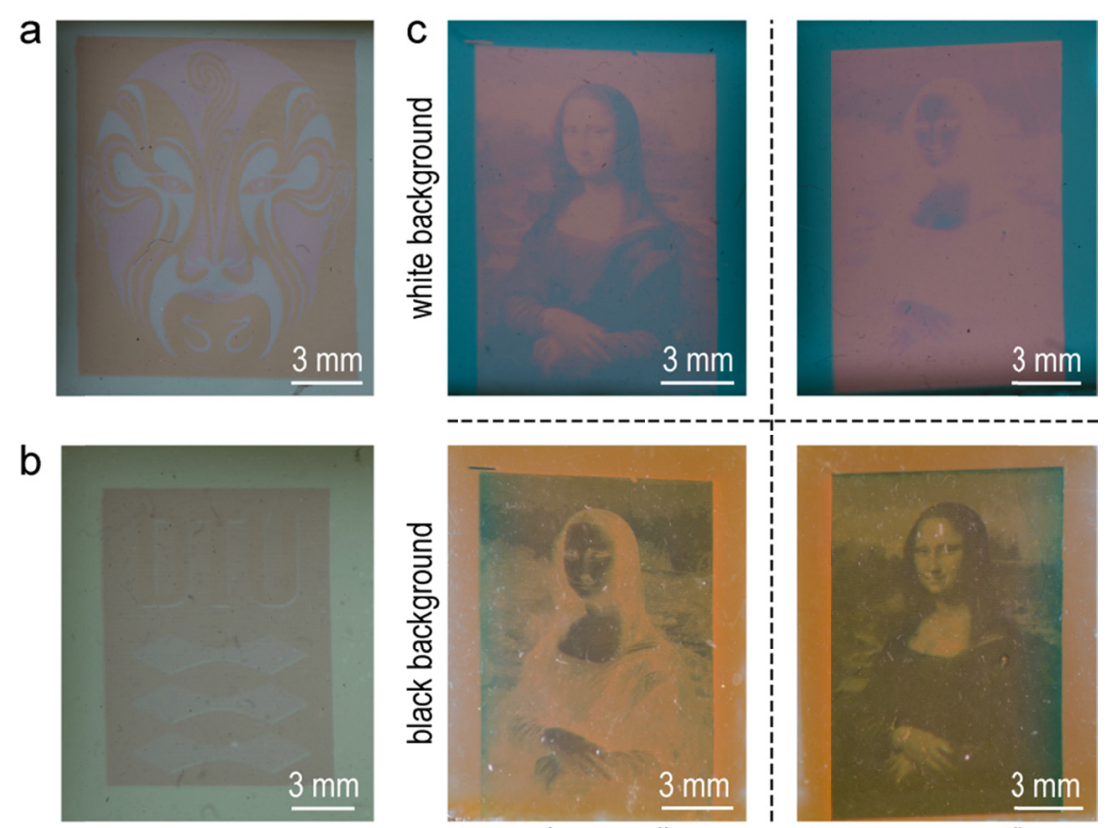

regular encoding

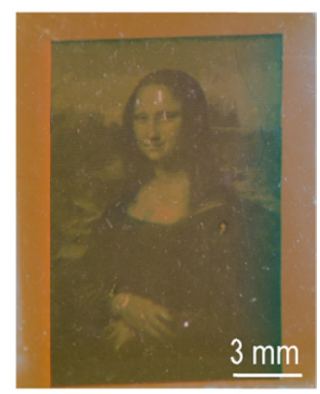

reverse encoding

Figure 4. Laser-printed stereoscopic images. a,b) Photographs of the laser-printed images in the different Au ND-embedded PDMS matrices. The samples are the matrix II (a) and another matrix embedded with the Au NDs that have an average diameter of $110 \mathrm{~nm}$ and thickness of $8 \mathrm{~nm}$ (b), respectively. c) Reflection (top) and transmission (bottom) halftoned images in the $\mathrm{Au}$ ND-embedded PDMS matrices IV with regular (left) and reverse (right) encoding, respectively.

The ability of stereoscopy is often preferred for laser printing, where not only various colors need to be expressed, but also printing at multiple depths needs to be realized. A 3D image can be mathematically divided into a series of two-dimensional color layers. As a proof-of-concept demonstration for our plasmonic color printing, three color layers were laser-printed at different depths by adjusting the focal plane position of the laser pulse at a step of $\sim 1 \mathrm{~mm}$. The transmission photograph of the matrix I with the color layers $1-3$ from front to back is displayed in Figure 5a. The overlapped region exhibits a darker shade with an increasing number of layers. In addition, the PDMS matrix was cut along the side of the layers 1 and 3 to examine the depths of the color layers (Figure $5 b$ ). To better identify each layer, the color layers 1-3 were printed with different laser pulse energies. The pulse energy applied on the color layers $1-3$ are $3.0 \mu \mathrm{J}, 0.6 \mu \mathrm{J}$ and $2.5 \mathrm{~nJ}$, respectively. According to the side views, the layer 1 is located near the top surface of the PDMS matrix while the layer 3 is embedded near the bottom surface. The thickness of each layer is estimated to be $\sim 1 \mathrm{~mm}$, 
indicating that bright colors can be laser-printed at a relatively low particle density $\left(6 \times 10^{15}\right.$ $\mathrm{m}^{-3}$ ) in the PDMS matrices. In principle, 3D stereoscopic color printing is possible by synergistically controlling the laser pulse energy and the focal plane. We note that a similar work on Au nanorod-embedded polyvinyl alcohol (PVA) matrices for optical date storage at multiple layers has been reported previously. ${ }^{[4]}$ Beyond the intrinsic properties of the NDs mentioned in the introduction, there are three more major differences between this previous work and our own. First, the previous work focuses on optical data recording, which requires the particle density to be several orders higher than that for our plasmonic color printing.

Second, the printing resolution in the previous work is restricted to a small region containing several particles while our plasmonic laser printing can be realized at the single-particle level. Third, the Au ND-embedded PDMS matrices are substrate-free and scalable in our work. The PVA matrices in the previous work need to be supported on substrates, with their sizes limited by the spin-coating technique.
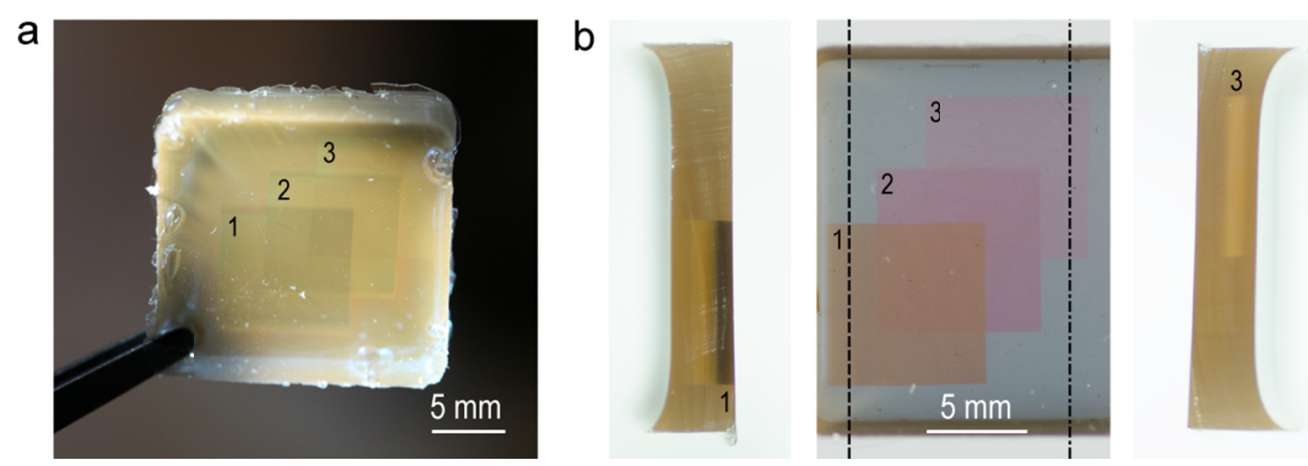

Figure 5. Multiple laser-printed color layers. a) Transmission photograph of the Au NDembedded PDMS matrix I with multiple laser-printed layers. The color layers 1-3 were printed with the laser pulse energy of $3.0 \mu \mathrm{J}, 0.6 \mu \mathrm{J}$ and $2.5 \mathrm{~nJ}$, respectively. b) Left side view (left), top view (middle) and right side view (right) of the cut PDMS matrix I. The dashed and dash-dotted lines indicate the cutting positions of the layers 1 and 3, respectively.

In summary, we have demonstrated the plasmonic color laser printing inside the transparent Au ND-embedded PDMS matrices. The uniform embedding of the circular $\mathrm{Au}$ NDs endows the transparent PDMS matrices with bright and tunable colors, which are economic, flexible and of long-term durability. During our laser printing process, both the 
morphological and spatial position of the Au NDs can be controlled in the 3D PDMS matrices to create plasmonic color images. We have achieved the full CMY color generation on the laser-printed stereoscopic images, which have a large area up to the centimeter scale and a resolution of 4600 DPI. The chromotropic characteristic of the printed Au ND-embedded PDMS matrices is also observed under reflected and transmitted light. Our work provides a robust method for the low-cost fabrication of large-area plasmonic patterns in 3D matrices. This technology paves the way for a variety of potential applications ranging from plasmonic color 3D display, security labeling, 3D-printing of optical components and data storage.

\section{Experimental Section}

Synthesis and PEGylation of the Circular Au NDs: The circular Au NDs were prepared by performing anisotropic oxidation on the hexagonal Au nanoplates that were synthesized using a seed-mediated method described previously. ${ }^{[32,42]}$ A mixture of $\mathrm{HCl}(1 \mathrm{M}, 240 \mu \mathrm{L})$ and $\mathrm{H}_{2} \mathrm{O}_{2}(30 \mathrm{wt} \%, 240 \mu \mathrm{L})$ was added into the as-prepared Au nanoplate solution $(40 \mathrm{~mL})$ whose extinction at the major plasmon peak of $\sim 935 \mathrm{~nm}$ was pre-adjusted to 3.0. After vigorous stirring, the mixture solution was kept in a $60{ }^{\circ} \mathrm{C}$ isothermal incubator to initiate the reaction. The oxidation process was monitored by measuring the extinction spectra of the reaction solution as a function of time. By adjusting the oxidation time, the circular Au NDs of different diameters can be synthesized. The reaction solution at an intermediate stage of oxidation was centrifuged and re-dispersed in deionized water, allowing for the production of the Au ND sample with a desired average diameter. The PEGylation process was carried out by adding aqueous mPEG-SH (RAPP Polymere, Germany, molecular weight: 5000, polymer chain concentration: $1 \mathrm{mM}, 0.5 \mathrm{~mL})$ into the as-grown Au ND solution (10 mL). After being left undisturbed overnight, the mixture solution was centrifuged to remove the excess mPEGSH and re-dispersed into ethanol $(0.5 \mathrm{~mL})$ for further use. 
Fabrication of the Circular Au ND-Embedded PDMS Matrices: The circular Au NDembedded PDMS matrices were fabricated according to the procedure described in our previous work. ${ }^{[36]}$ Typically, the ethanolic PEGylated Au ND solution $(0.5 \mathrm{~mL})$ was first mixed with the PDMS prepolymer component A (5 g). The mixture of the Au NDs and the PDMS prepolymer was continuously stirred under ultrasonication until it exhibited a uniform color. The PDMS component B (0.5 g) was then added for crosslinking. The mixture was thereafter filled into a mold and left undisturbed at room temperature. After the mixture became transparent, the mold was heated up to and kept at $65^{\circ} \mathrm{C}$ for $1 \mathrm{~h}$, allowing for the polymerization of the PDMS prepolymer. By applying the circular Au ND samples with different average diameters, the Au ND-embedded PDMS matrices with controllable colors were produced.

Optical Measurements: The extinction spectra were acquired on a PerkinElmer Lambda 950 ultraviolet/visible/near-infrared spectrophotometer. The aqueous Au ND samples were measured with plastic cuvettes of $1.0 \mathrm{~cm}$ in optical path length. For the Au ND-embedded PDMS matrices, blank PDMS matrices with the same thicknesses were employed as the references. The single-particle dark-field scattering spectra were recorded on an Olympus BX60 upright microscope that was composed of a quartz-tungsten-halogen lamp $(100 \mathrm{~W})$, a monochromator (Acton, SpectraPro 2360i), and a charge-coupled device camera (Princeton Instruments, Pixis 400 , cooled to $-70^{\circ} \mathrm{C}$ ). A $100 \times$ dark-field objective (numerical aperture: 0.9) was used for both the excitation of the individual nanoparticles with the white light and the collection of the scattered light. The laser printing was performed on a Nikon Ti-U inverted microscope, where the pulses ( $1 \mathrm{~ns}, 532 \mathrm{~nm}$ ) from a laser (CryLaS FDSS532-150) were focused with a $4 \times$ bright-field objective (numerical aperture: 0.13 ). The laser pulse energy was precisely controlled and rapidly modulated with a liquid-crystal attenuator. The Au ND-embedded PDMS matrix was mounted on a computer-controlled piezo nanopositioning stage (Mad City Labs, Nano-H50 series piezoelectric stage, $0.1 \mathrm{~nm}$ resolution, 
$50 \mathrm{~mm}$ travel distance, $\mathrm{XY}$ axes). The photographs were acquired using a commercial Nikon D7000 camera.

Finite-Difference Time-Domain Simulations: The FDTD simulations were performed using FDTD Solutions 8.7 developed by Lumerical Solutions. During the simulations, an electromagnetic pulse in the spectral range from $400 \mathrm{~nm}$ to $1400 \mathrm{~nm}$ was launched into a box containing a target nanostructure. A mesh size of $1 \mathrm{~nm}$ was employed in calculating the scattering and absorption spectra. The Au NDs, nanospheres and nanorod were modelled as a cylinder, a sphere and a cylinder with two hemispherical ends, respectively. The geometrical parameters of the NDs were set according to the measured average values. For simplicity, the glass-supported Au spheroidal nanoparticles were approximated as spherical nanoparticles with gradually decreasing diameters during the simulations. For the comparison of the absorption and scattering cross-sections among the three differently shaped Au nanocrystals, the particle volumes of the nanosphere and nanorod were set to be equal to that of the ND, and the longitudinal dipolar plasmon wavelength of the nanorod was set to be equal to that of the in-plane dipolar plasmon wavelength of the ND by adjusting the diameter and length of the nanorod. The three nanocrystals were immersed in water. The refractive indexes of air, water, PDMS and cover glass were set at 1.0, 1.33, 1.4 and 1.45, respectively. The dielectric function of gold was taken from Johnson and Christy's data.

\section{Supporting Information}

Supporting Information is available from the Wiley Online Library or from the author.

\section{Acknowledgements}

X.M.C. and X.L.Z. contributed equally to this work. J.F.W. and A.K. conceived and supervised the project. X.M.C., X.L.Z. and L.S. performed the experiments. X.M.C. and L.S. carried out the synthesis of the nanocrystal-embedded PDMS matrices. X.L.Z. built the optical setup and implemented the laser printing. X.M.C. performed the sample characterization and the FDTD simulations. All authors discussed the results and contributed to the writing of the manuscript. The authors thank S. Raza for fruitful discussion, W. Z. Lu and J. Scheel for help with taking the photographs. The authors acknowledge the financial support from the Danish-Chinese international network programme PLASMONCOLOR (Grant No.: 6144-00047). J.F.W., X.M.C. and L.S. acknowledge the financial support from 
the Hong Kong Research Grants Council (GRF, 14306817) and CUHK Direct Grant (2017-18, Project Code: 4053307). X.L.Z. acknowledges the support of VILLUM Experiment (Grant No.: 17400) from VILLUM FONDEN. A.K. and X.L.Z. were also supported by the European Commission through the H2020 FET-OPEN project CHROMAVISION (Grant Agreement No.: 665233).

Received: ((will be filled in by the editorial staff))

Revised: ((will be filled in by the editorial staff)) Published online: ((will be filled in by the editorial staff))

References

[1] I. Freestone, N. Meeks, M. Sax, C. Higgitt, Gold Bull. 2007, 40, 270.

[2] A. Kristensen, J. K. W. Yang, S. I. Bozhevolnyi, S. Link, P. Nordlander, N. J. Halas, N. A. Mortensen, Nat. Rev. Mater. 2016, 2, 16088.

[3] N. Dean, Nat. Nanotechnol. 2015, 10, 15.

[4] D. K. Gramotnev, S. I. Bozhevolnyi, Nat. Photonics 2010, 4, 83.

[5] H. J. Chen, L. Shao, Q. Li, J. F. Wang, Chem. Soc. Rev. 2013, 42, 2679.

[6] K. Kumar, H. G. Duan, R. S. Hegde, S. C. W. Koh, J. N. Wei, J. K. W. Yang, Nat. Nanotechnol. 2012, 7, 557.

[7] A. S. Roberts, A. Pors, O. Albrektsen, S. I. Bozhevolnyi, Nano Lett. 2014, 14, 783.

[8] A. S. Roberts, S. M. Novikov, Y. Q. Yang, Y. T. Chen, S. Boroviks, J. Beermann, N. A. Mortensen, S. I. Bozhevolnyi, ACS Nano 2019, 13, 71.

[9] T. Kraus, L. Malaquin, H. Schmid, W. Riess, N. D. Spencer, H. Wolf, Nat. Nanotechnol. 2007, 2, 570 .

[10] X. L. Zhu, W. Yan, U. Levy, N. A. Mortensen, A. Kristensen, Sci. Adv. 2017, 3, e1602487.

[11] X. L. Zhuo, H. K. Yip, X. M. Cui, J. F. Wang, H.-Q. Lin, Light: Sci. Appl. 2019, 8, 39.

[12] M. Ozaki, J.-I. Kato, S. Kawata, Science 2011, 332, 218.

[13] K. T. P. Lim, H. L. Liu, Y. J. Liu, J. K. W. Yang, Nat. Commun. 2019, 10, 25.

[14] L. Shao, X. L. Zhuo, J. F. Wang, Adv. Mater. 2018, 30, 1704338. 
[15] X. Y. Duan, S. Kamin, N. Liu, Nat. Commun. 2017, 8, 14606.

[16] T. Xu, Y.-K. Wu, X. G. Luo, L. J. Guo, Nat. Commun. 2010, 1, 59.

[17] T. Ellenbogen, K. Seo, K. B. Crozier, Nano Lett. 2012, 12, 1026.

[18] X. M. Goh, Y. H. Zheng, S. J. Tan, L. Zhang, K. Kumar, C.-W. Qiu, J. K. W. Yang, Nat. Commun. 2014, 5, 5361.

[19] S. J. Tan, L. Zhang, D. Zhu, X. M. Goh, Y. M. Wang, K. Kumar, C.-W. Qiu, J. K. W. Yang, Nano Lett. 2014, 14, 4023.

[20] S. Yokogawa, S. P. Burgos, H. A. Atwater, Nano Lett. 2012, 12, 4349.

[21] F. Cheng, J. Gao, L. Stan, D. Rosenmann, D. Czaplewski, X. D. Yang, Opt. Express 2015, 23, 14552 .

[22] X. L. Zhu, C. Vannahme, E. Højlund-Nielsen, N. A. Mortensen, A. Kristensen, Nat. Nanotechnol. 2016, 11, 325.

[23] J.-M. Guay, A. C. Lesina, G. Côté, M. Charron, D. Poitras, L. Ramunno, P. Berini, A. Weck, Nat. Commun. 2017, 8, 16095.

[24] A. Habenicht, M. Olapinski, F. Burmeister, P. Leiderer, J. Boneberg, Science 2005, 309, 2043.

[25] D. A. Zuev, S. V. Makarov, I. S. Mukhin, V. A. Milichko, S. V. Starikov, I. A. Morozov, I. I. Shishkin, A. E. Eransnok, P. A. Belov, Adv. Mater. 2016, 28, 3087.

[26] L. Shao, Y. T. Tao, Q. F. Ruan, J. F. Wang, H.-Q. Lin, Phys. Chem. Chem. Phys. 2015, $17,10861$.

[27] T. Siegfried, Y. Ekinci, O. J. F. Martin, H. Sigg, ACS Nano 2013, 7, 2751.

[28] J. S. Clausen, E. Højlund-Nielsen, A. B. Christiansen, S. Yazdi, M. Grajower, H. Taha, U. Levy, A. Kristensen, N. A. Mortensen, Nano Lett. 2014, 14, 4499.

[29] E. Højlund-Nielsen, J. Clausen, T. Mäkela, L. H. Thamdrup, M. Zalkovskij, T. Nielsen, N. L. Pira, J. Ahopelto, N. A. Mortensen, A. Kristensen, Adv. Mater. Technol. 2016, 1, 160054. 
[30] Y. N. Xia, Y. J. Xiong, B. Lim, S. E. Skrabalak, Angew. Chem. Int. Ed. 2009, 48, 60.

[31] T. K. Sau, A. L. Rogach, F. Jäckel, T. A. Klar, J. Feldmann, Adv. Mater. 2010, 22, 1805.

[32] X. M. Cui, F. Qin, Q. F. Ruan, X. L. Zhuo, J. F. Wang, Adv. Funct. Mater. 2018, 28, 1705516.

[33] P. Hanarp, M. Käll, D. S. Sutherland, J. Phys. Chem. B 2003, 107, 5768.

[34] M. N. O’Brien, M. R. Jones, K. L. Kohlstedt, G. C. Schatz, C. A. Mirkin, Nano Lett. 2015, 15,1012 .

[35] A. Mendoza-Galván, K. Järrendahl, A. Dmitriev, T. Pakizeh, M. Käll, H. Arwin, Opt. Express 2011, 19, 12093.

[36] C. H. Fang, L. Shao, Y. H. Zhao, J. F. Wang, H. K. Wu, Adv. Mater. 2012, 24, 94.

[37] A. Lamberti, A. Virga, P. Rivolo, A. Angelini, F. Giorgis, J. Phys. Chem. B 2015, 119, 8194.

[38] Y. N. Xia, G. M. Whitesides, Angew. Chem. Int. Ed. 1998, 37, 550.

[39] J. A. Rogers, T. Someya, Y. G. Huang, Science 2010, 327, 1603.

[40] F. Kotz, K. Plewa, W. Bauer, N. Schneider, N. Keller, T. Nargang, D. Helmer, K.

Sachsenheimer, M. Schäfer, M. Worgull, C. Greiner, C. Richter, B. E. Rapp, Adv. Mater. 2016, 28,4646

[41] M. M. Ito, A. H. Gibbons, D. Qin, D. Yamamoto, H. D. Jiang, D. Yamaguchi, K. Tanaka, E. Sivaniah, Nature 2019, 570, 363

[42] F. Qin, T. Zhao, R. B. Jiang, N. N. Jiang, Q. F. Ruan, J. F. Wang, L.-D. Sun, C.-H. Yan, H.-Q. Lin, Adv. Opt. Mater. 2016, 4, 76.

[43] S. Kawata, H.-B. Sun, T. Tanaka, K. Takada, Nature 2001, 412, 697.

[44] W. L. Barnes, A. Dereux, T. W. Ebbesen, Nature 2003, 424, 824.

[45] P. Zijlstra, J. W. M. Chon, M. Gu, Nature 2009, 459, 410. 


\section{Table of Contents Entry}

A robust laser printing method is realized for the low-cost fabrication of large-area plasmonic patterns in three-dimensional transparent matrices. Colloidal circular gold nanodisks are uniformly embedded inside poly(dimethylsiloxane) matrices with tailorable plasmonic colors. Both the morphological and spatial position of the embedded gold nanodisks can be finely manipulated through a stereo scanning of the laser spot.

Keyword

Plasmonic Color Laser Printing

Ximin Cui, Xiaolong Zhu, Lei Shao, Jianfang Wang, ${ }^{*}$ and Anders Kristensen*

Plasmonic color laser printing inside transparent gold nanodisk-embedded poly(dimethylsiloxane) matrices

ToC figure

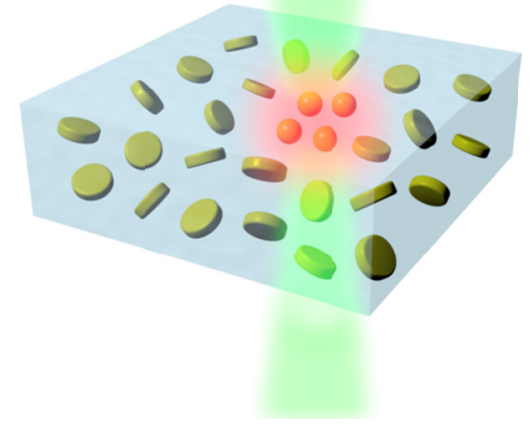

